

\title{
Product differentiation in successive vertical oligopolies
}

\author{
Paul Belleflamme* $\quad$ Eric Toulemonde ${ }^{\dagger}$
}

October 2000

\begin{abstract}
This is a successive oligopoly model with two brands. Each downstream firm chooses one brand to sell on a final market. The upstream firms specialize in the production of one input specifically designed for the production of one brand, but they also produce the input for the other brand at an extra cost. We show that when more downstream firms choose one brand, more upstream firms will specialize in the input specific to that brand, and vice versa. Hence, multiple equilibria are possible and the softening effect of brand differentiation on competition might not be strong enough to induce maximal differentiation. The existence of equilibria and their welfare performance are also examined.
\end{abstract}

JEL classification codes: L11, L13, L23

Keywords: product differentiation, vertical relationships, oligopoly

*Department of Economics, Queen Mary, University of London, Mile End Road, London E1 4NS, United Kingdom (e-mail: p.belleflamme@qmw.ac.uk, phone: + 44207882 5587, fax: +44 208983 3580, home page: http://www.qmw.ac.uk/ ugte186/)

${ }^{\dagger}$ Chargé de recherche at the National Fund for Scientific Research (Belgium), C.R.E.W., G.R.E.B.E., Department of Economics, University of Namur, 8 Rempart de la Vierge, 5000 Namur, Belgium (e-mail: eric.toulemonde@fundp.ac.be, phone: + 3281 724810, fax: +32 81 724840, home page: http://www.fundp.ac.be/ etoulemo). 


\section{Introduction}

Since the pioneering work of Hotelling (1929), the softening effect of product differentiation on price competition has been abundantly studied. For instance, d'Aspremont et al. (1979) establish that firms choose maximal differentiation in the linear city model with quadratic transportation costs. This prediction is echoed in most marketing texts concerning market segmentation, which recommend firms to differentiate their products.

There exist, however, forces that oppose maximal (or any) product differentiation. Following Tirole (1988), these forces can be put into three categories. A first, obvious, category has to do with the absence of price competition: in some instances, there may exist legal or technical reason why the scope of price competition is limited. The second category corresponds to the motto "Be where the demand is". Although firms like to differentiate for strategic purposes, they also all want to locate where the demand is. If, for example, demand is concentrated around a few poles, one can easily construct examples in which firms differentiate but not fully. In a similar vein, search by consumers may encourage firms to gather. Finally, the third category refers to positive externalities that induce firms to locate near one another. The now standard classification of Marshallian externalities is between localization economies (which refer to the benefits generated by the proximity of firms producing similar goods), and urbanization economies (which account for all the advantages associated with the overall level of activity prevailing in a particular area). The general idea is that, for given inputs, the output of an individual firm is larger the larger is the aggregate output of other firms producing the same good in the same locale.

Regarding the latter category, Helpman and Krugman (1985) point out that Marshall (1920)'s explanation is incomplete: external economies can arise from proximity to specialized inputs only if there is a natural comparative advantage for the production of these inputs in the region. As Rotemberg and Saloner (2000) nicely put it, "The puzzle is simply rolled back to the previous production stage: Why do the producers of inputs locate in the region?". They develop therefore the theory that the equilibrium locations of firms and their input suppliers are interdependent. So does Venables (1996) who argues:

"If industries are vertically linked through an input-output structure, 
then the downstream industry forms the market for upstream. Market access considerations then draw the upstream industry to locations where there are relatively many downstream firms. In addition to this demand linkage between industries, there is also a cost linkage. Firms in the downstream industry will have lower costs if they locate where there are relatively many upstream firms-they save trade costs on their intermediate inputs. Putting the demand linkage and the cost linkage together creates a force for the agglomeration of activity in a single location."

The present paper shares the intuition of Venables (1996) but departs from his analysis in one important aspect. Venables is concerned with regional agglomeration and trade, and considers thus physical location of firms in different regions. By contrast, we address the issue of product differentiation, and consider thus location of firms in a product rather than in a geographical space. Where Venables considers exogenously differentiated products which can be sold on two different geographical markets, we focus on a single market on which two endogenously differentiated products can be sold. Our main objective is thus to assess how the softening effect of product differentiation on competition might be mitigated by the demand and cost linkages identified by Venables.

Specifically, we consider two vertically related industries with the following features. The downstream industry produces a final product that can be marketed under two possible differentiated brands. Intuitively, one understands that, absent any other consideration, this industry is driven towards a situation where firms split equally (or 'almost equally' if there is an odd number of them) between the two brands in order to soften competition (this is the translation of maximal differentiation in our setting).

However, this intuition may prove wrong when we also take into account an upstream industry that produces an essential input for the downstream industry, and that also has to take its stand on the two brands. The reason is the following. Although the use of so-called flexible manufacturing systems $(F M S)$ becomes increasingly widespread, ${ }^{1}$ economies of scale are still present in many industries. It is thus reasonable to assume that the input is more

\footnotetext{
${ }^{1}$ As explained by Norman and Thisse (1999), "the essence of a FMS is that it allows firms to customize their products to the requirements of heterogeneous customers at little or no cost penalty".
} 
costly to produce when it has to fit two differentiated brands rather than a single one. This induces suppliers to select the brand from which they want to "get closer". Specifically, suppliers will incur specific costs to adapt the intermediate product to the specific needs of a single brand of the final product. Nevertheless, such specific investment does not completely prevent them to also conform their input to the other brand: depending on the demand expressed by the buyers of this other brand, they might indeed find it profitable to incur the extra cost to serve them. Adopting the terminology of Eaton and Schmitt (1994), we can say equivalently that upstream firms choose to develop one basic product (that fits one particular brand of the final good), and then produce, with an extra cost, one variation of that basic product (in order to fit the other brand of the final good). ${ }^{2}$

Reconsidering brand choices by downstream firms in such a broader context, we can conjecture that these choices will be driven not only by competition on the final market, but also by cost considerations linked to the choices made by firms in the upstream industry. In particular, downstream firms will be more attracted by a brand that a large number of upstream firms have chosen to conform with. Since, in turn, upstream firms are more likely to conform with a brand that has been selected by a large number of downstream firms, we are in the presence of mutually re-enforcing forces that drive the industries toward an absence of product differentiation.

Our goal is to analyze the balance between these various forces in a simple static two-stage model where, first, firms in both industries choose simultaneously between the two brands and, second, compete on their respective markets. We show that the number of downstream firms that choose one particular brand increases with the number of upstream firms that specialize in the input that is specific to that brand, and vice versa. This raises the possibility of multiple equilibria. For instance, it is possible to have an equilibrium without any differentiation (upstream and downstream firms select the same brand), or an equilibrium with maximal differentiation (firms split equally between both brands) or an intermediate equilibrium (most-but not all-upstream and downstream firms choose the same brand). Depending on parameters, some of these equilibria are preferred by firms while the consumer prefer another equilibrium. Hence, there is no clear-cut conclusion to

\footnotetext{
${ }^{2}$ This extra cost might be due to the fact that more raw material is needed for each unit of the input to be transformed into the other brand. It might also be the consequence of a degradation of performance of the input when it is transformed into the other brand.
} 
draw for society as a whole, which might end up with excessive or insufficient differentiation.

We see two other possible applications of our setting. First, the extra cost incurred by the upstream firms might follow from other sources than the necessity to physically alter the basic product. It might well be the case that suppliers face different costs for serving different buyers, even though they sell them exactly the same physical good. For instance, the development of EDI (Electronic Data Interchange) often induces suppliers to conduct transactions in different ways with different buyers. ${ }^{3}$ As explained by Angeles and Nath (2000), "with the movement towards more integrated and agile 'extended enterprises', hub firms (i.e. firms that initiate EDI linkages) have been forced to provide incentives to their suppliers to make non-contractible investments in information sharing, quality initiatives, and innovation to enable them to fulfill the requirements of more tightly connected and integrated information networks." Similarly, case studies in Marcussen (1996) show that EDI capability influences the buying decision about which upstream firms will be 'in suppliers' (currently chosen suppliers) - and which of the 'in suppliers' will get orders for specific items. "If two leading competing suppliers both develop EDI capability, competitive parity between the two firms is maintained. However, the EDI capable 'in suppliers' stand to win at the expense of both the 'in suppliers' without EDI capability and the 'out suppliers"'. Since EDI requirements often differ from one downstream firm to another, suppliers willing to trade with different buyers face the same type of costs as the ones described above.

Second, the issue we address in this paper can also be related to the literature considering mix-and-match and system goods. As defined by Einhorn (1992), "mix-and-match compatible technologies are systems of interconnected goods that may incorporate different components from different manufacturers". Examples abound, e.g., in the computer and in the consumer electronics industries. Some papers in this literature consider industries where each firm sells every component of a complete system. ${ }^{4}$ Other papers consider instead that firms specialize in the production of a single component, an assumption that comes closer to the issue at stake here (we

\footnotetext{
${ }^{3} \mathrm{EDI}$ is the direct computer-to-computer exchange of information stored in standard formatted business documents, such as invoices, bills of lading, purchase orders, etc., among firms.

${ }^{4}$ See,e.g., Matutes and Régibeau (1988, 1992), Economides (1989).
} 
can indeed interpret our intermediate and final products as complementary components forming a system good). Although the papers in this second strand of the literature focus on different issues than ours (and make quite different assumptions about, notably, consumer preferences and 'compatibility' costs), they share our concern for analyzing the "location" decisions of the 'software' firms (the upstream firms in our terminology). ${ }^{5}$ However, it must be stressed that they leave aside the "location" decisions of the 'hardware' (or downstream) firms and, therefore, abstract away our main question about how the decisions of both types of firms affect each other.

The rest of the paper is organized as follows. Section 2 describes the modelling framework. Section 3 draws some intuition from two simple models where costs are taken as exogenous. Section 4 analyzes the general twostage game and presents our main results. Section 5 collects some welfare considerations. Section 6 concludes and proposes some directions for further research.

\section{The model}

The model is a successive oligopoly model with two adjacent industries. In the downstream industry, a set $N$ of $n$ firms produce some product to be sold on a final market. They all have to choose under which brand to market this product. Two horizontally differentiated brands, noted $a$ and $b$, are available. Once the firms have made their choice, the industry is partitioned into the two subsets $N_{a}$ and $N_{b}\left(N_{a} \cap N_{b}=\emptyset, N_{a} \cup N_{b}=N\right)$. We adopt the following notation. Let $y_{i k}$ denote the quantity of brand $k(k=a, b)$ produced by some downstream firm $i(i=1, \ldots, n), Y_{k} \equiv \sum_{i \in N_{k}} y_{i k}$, the total quantity produced of brand $k$, and $Y_{k}^{-i} \equiv \sum_{j \in N_{k}, j \neq i} y_{j k}$.

Within the existing theory, there are two basic approaches to model horizontal differentiation. On the one hand, the spatial models-such as the

\footnotetext{
${ }^{5}$ For instance, Matutes and Régibeau (1989) analyze the situation where two firms sell one component (e.g., software) of a two-component system, and where consumers have already bought the first component (e.g., the personal computer) so that several independent markets for software are created. Firms have thus to choose the market(s) that they will serve. They also decide whether to produce different software for each market or to sell software that can be used with both types of computers. Church and Gandal (1992) develop a model where there are two incompatible hardware technologies, an endogenously determined number of software firms, and consumers who value software variety; they investigate the decision of a software firm concerning which network to join.
} 
Hotelling's linear city and Salop's circular city models-generate market demands by integrating over consumers with different tastes. On the other hand, the models in the Spence-Dixit-Stiglitz tradition derive a demand system for differentiated products from the utility function of a representative consumer with a taste for variety. ${ }^{6}$ Because the first approach is based on a rather simplistic description of individual demand (consumers are assumed to buy at most one unit of the product), it proves ill-suited to incorporate demand and cost linkages. We thus favor the second approach and use a system of demand functions for differentiated products derived from the quadratic, separable utility function of a representative consumer, as in Shubik (1980, Chapter 7) or in Singh and Vives (1984). Accordingly, we write the inverse demand schedule as follows: ${ }^{7}$

- when the $n$ downstream firms choose to produce the same brand $k$ (meaning that $N_{k}=N$ and that the other brand is not produced), price on the market for brand $k$ is given by $p_{k}=1-Y_{k}$;

- when the downstream firms choose to produce different brands, prices on the market are given by $p_{a}=1-Y_{a}-\gamma Y_{b}$ and $p_{b}=1-Y_{b}-\gamma Y_{a}$ in the region of quantities where prices are positive, where $0 \leq \gamma<1$ is the degree of differentiation between brands $a$ and $b$.

We assume that the downstream firms produce the final product by transforming a single intermediate good on a one-for-one basis. The marginal cost of the downstream firms only consists of the price paid for the intermediate good (other specific costs are assumed to be zero).

The intermediate good is supplied by the upstream industry, which consists of a set $M$ of $m$ firms. The production technology for the intermediate good assumes some degree of 'brand specificity'. That is, each upstream firm has to design its production process in conformity with a specific brand of the final product (we say in the sequel that they choose to "produce for" brand $a$ or for brand $b$ ). As a result, if the intermediate good is to be transformed into that specific brand, the marginal cost of production is equal to

\footnotetext{
${ }^{6}$ For a recent discussion of these two approaches (and the presentation of a novel integrative approach), see Anderson and de Palma (2000).

${ }^{7}$ The inverse demand schedule is derived from the quadratic utility function of a representative consumer which exhibits love for variety; for the exact derivation, see the proof of Proposition 7 in the appendix.
} 
$c$, whereas if it has to be transformed into the other brand, an extra 'adaptation' cost $t$ is to be incurred, and the total marginal cost of production amounts to $c+t$ (naturally, we assume $c+t<1$ to avoid no trade situations). ${ }^{8}$ In the sequel, without any loss of generality, we set $c=0$. For the sake of simplicity, we also assume that both industries count an even number of firms (in Subsection 4.4, we discuss how an odd number of firms in either industry affects our main results).

We study a dynamic game where location decisions (which brand to produce, or to design the intermediate good for?) precede production decisions (how much of the intermediate and the final goods to produce?). Regarding production decisions, we assume a sequential Cournot competition in which upstream firms decide before downstream firms, the price of the intermediate good being obtained by equating supply to demand. Regarding brand choices, we assume simultaneous decisions by upstream and downstream firms. The game is solved for its subgame-perfect equilibria by the method of backward induction.

Although the modelling framework has been kept as simple as possible, the dynamic game is rather intricate to solve. Therefore, to shed some light on the various forces at play, we shall first focus on the downstream industry and analyze two simple models where costs are taken as exogenous. This will allow us to better grasp how downstream decisions are affected by the endogeneization of costs through the upstream brand choices.

\section{Brand choices with exogenous costs}

We start with the second-stage production decisions. Suppose that the two brands have been adopted by a positive number of firms. Typical firms $i \in N_{a}$ and $j \in N_{b}$ respectively face the following maximization programs:

\footnotetext{
${ }^{8}$ A concrete example that fits our assumptions is what Levitt (1980) reports about a specific type of steel, the so-called 'No. 302, 72-inch, hot-rolled strip'. "Not all generic products are the same. (...) Because of slight differences among automobile company manufacturing processes, one supplier's "302" may, in fact, be "better" than another's. One mill's 302 may take certain coatings more easily or quickly than another's. One supplier may fill orders from a single mill, and another from several. In the latter case, the sheen or hue of the generic product may vary slightly from mill to mill, which makes considerable difference in the case of stainless steel that is used for decorative trim."
} 


$$
\begin{aligned}
\max _{y_{i a}} \Pi_{i}^{d} & =\left(1-y_{i a}-Y_{a}^{-i}-\gamma Y_{b}\right) y_{i a}-w_{a} y_{i a}, \\
\max _{y_{j b}} \Pi_{j}^{d} & =\left(1-y_{j b}-Y_{b}^{-j}-\gamma Y_{a}\right) y_{j b}-w_{b} y_{j b},
\end{aligned}
$$

where $w_{a}$ and $w_{b}$ are the prices of the intermediate input to be transformed in either brand $a$ or $b$. For the moment, we assume that these prices are constant.

The first-order conditions for profit maximization yield the following reaction functions: $y_{i a}=(1 / 2)\left(1-w_{a}-Y_{a}^{-i}-\gamma Y_{b}\right)$ and $y_{j b}=(1 / 2)(1-$ $\left.w_{b}-Y_{b}^{-j}-\gamma Y_{a}\right)$. Solving this system and using the symmetric positions of firms within each market, we derive the equilibrium quantities. To simplify the exposition, we identify a partition of the firms to $\Delta_{n} \equiv n_{a}-n_{b}$ where $n_{k}=\# N_{k}, k=a, b$. For a given $\Delta_{n}$ inherited from the first stage, every $i \in N_{a}$ produce a quantity $y_{a}\left(\Delta_{n}\right)$, and every $j \in N_{b}$ a quantity $y_{b}\left(\Delta_{n}\right)$ :

$$
\begin{aligned}
& y_{a}\left(\Delta_{n}\right)=2 \frac{\left(n-\Delta_{n}+2\right)\left(1-w_{a}\right)-\gamma\left(n-\Delta_{n}\right)\left(1-w_{b}\right)}{4(n+1)+\left(1-\gamma^{2}\right)\left(n^{2}-\Delta_{n}^{2}\right)}, \\
& y_{b}\left(\Delta_{n}\right)=2 \frac{\left(n+\Delta_{n}+2\right)\left(1-w_{b}\right)-\gamma\left(n+\Delta_{n}\right)\left(1-w_{a}\right)}{4(n+1)+\left(1-\gamma^{2}\right)\left(n^{2}-\Delta_{n}^{2}\right)} .
\end{aligned}
$$

In the case where all firms adopt the same brand (say brand $a$ ), we set $\Delta_{n}=n$ in expression (1) and disregard expression (2). ${ }^{9}$ Equilibrium profits are simply equal to the square of the equilibrium quantities: $\Pi_{a}^{d}\left(\Delta_{n}\right)=$ $\left[y_{a}\left(\Delta_{n}\right)\right]^{2}$ and $\Pi_{b}^{d}\left(\Delta_{n}\right)=\left[y_{b}\left(\Delta_{n}\right)\right]^{2}$.

Let us now turn to the first stage of the game where firms simultaneously choose which brand they want to produce. In this game, a Nash equilibrium with $\Delta_{n} \neq \pm n$ is characterized by two conditions, ensuring that no firm finds it profitable to switch brands unilaterally: (Da) $\Pi_{a}^{d}\left(\Delta_{n}\right) \geq \Pi_{b}^{d}\left(\Delta_{n}-2\right)$ and (Db) $\Pi_{b}^{d}\left(\Delta_{n}\right) \geq \Pi_{a}^{d}\left(\Delta_{n}+2\right)$. A Nash equilibrium with $\Delta_{n}=n$ (resp. $\Delta_{n}=-n$ ) satisfies (Da) (resp. (Db)) only. We develop these conditions in two specific examples.

\subsection{Constant and identical costs}

In the first example, we assume that all firms face the same marginal cost, whatever their choice of brand. For $w_{a}=w_{b}=w$, conditions (Da) and (Db)

\footnotetext{
${ }^{9}$ We suppose that parameter values are such that equilibrium quantities are positive for all $\Delta_{n}$ (this issue is analyzed rigorously in the general setting).
} 
can respectively be rewritten as

$$
\left\{\begin{array}{l}
(\mathbf{D a})-\left(\Delta_{n}-1\right)\left[4(n-\gamma)+\left(1-\gamma^{2}\right)\left(n-\Delta_{n}\right)\left(n+\Delta_{n}-2\right)\right] \geq 0, \\
(\mathbf{D b})-\left(-\Delta_{n}-1\right)\left[4(n-\gamma)+\left(1-\gamma^{2}\right)\left(n+\Delta_{n}\right)\left(n-\Delta_{n}-2\right)\right] \geq 0 .
\end{array}\right.
$$

Because condition (Da) [resp. (Db)] is valid for $-n<\Delta_{n} \leq n$ [resp. $\left.-n \leq \Delta_{n}<n\right]$, it is clear that the two bracketed terms are strictly positive, meaning that the two conditions boil down to $-1 \leq \Delta_{n} \leq 1$. In words, when all downstream firms face the same cost of production, the unique brand choice equilibrium is where firms split equally between the two brands $\left(\Delta_{n}=0\right) .{ }^{10}$ The intuition is simple: when the split is unequal, firms on the 'larger' market have an incentive to move to the 'smaller' market where competition is softer.

\subsection{Exogenous Marshallian externalities}

In the second example, we introduce exogenous Marshallian externalities of the following linear form: $w_{k}=w-\theta n_{k}$, with $0<\theta<w / n$, and $k=a, b$. We assume thus that the marginal cost of producing some brand decreases linearly with the number of firms producing that particular brand (see Section 1 for justifications of such assumption). In this case, we can use expressions (1) and (2) to develop conditions (Da) and (Db) as follows:

$$
\left\{\begin{array}{lll}
\text { (Da) } & \left(\Delta_{n}-1\right)[\theta(1+\gamma n)-(1-\gamma)(1-w)] & \left(\Delta_{n}\right) \geq 0 \\
\text { (Db) }\left(\Delta_{n}+1\right)[\theta(1+\gamma n)-(1-\gamma)(1-w)] & \left(\Delta_{n}+2\right) \leq 0
\end{array}\right.
$$

where $\quad\left(\Delta_{n}\right)=\left[(n(1-\gamma)+2)(n(1+\gamma)-2 \gamma)-\left(1-\gamma^{2}\right) \Delta_{n}\left(\Delta_{n}-2\right)\right]$. It can be shown that $\left(\Delta_{n}\right)>0$, for all $-n+2 \leq \Delta_{n} \leq n .{ }^{11}$ There are therefore two cases to consider according to the intensity of the Marshallian externalities. We define the following threshold: $\bar{\theta} \equiv(1-\gamma)(1-w) /(1+\gamma n)$.

1. For weak Marshallian externalities $(\theta \leq \bar{\theta})$, conditions (Da) and (Db) boil down to $-1 \leq \Delta_{n} \leq 1$, meaning that the previous conclusion carries over: equal split is the unique brand choice equilibrium.

\footnotetext{
${ }^{10}$ When $n$ is odd, there are two equilibria, $\Delta_{n}=1$ and $\Delta_{n}=-1$, which correspond to an 'almost equal' split of the set of firms (see Subsection 4.4 for more on this issue).

${ }^{11}$ See the proof of Lemma 1 in the Appendix.
} 
2. For strong Marshallian externalities $(\theta>\bar{\theta})$, conditions (Da) and (Db) cannot hold at the same time. There is thus no brand choice equilibrium where the two brands are both chosen by a positive number of firms. The only equilibria are where all firms choose the same brand.

Again, the intuition is simple: when Marshallian externalities are strong enough, they overcome the competitive attractiveness of the 'smaller' market and drive the industry towards concentration on a single brand.

\section{Brand choices with endogenous costs}

We now explicitly consider the upstream industry in order to endogenize the downstream input costs. Using the downstream reaction functions derived above, we obtain the inverse demands for the intermediate good to be transformed in each brand:

$$
\begin{aligned}
& w_{a}=1-\frac{n+\Delta_{n}+2}{n+\Delta_{n}} Y_{a}-\gamma Y_{b}, \\
& w_{b}=1-\frac{n-\Delta_{n}+2}{n-\Delta_{n}} Y_{b}-\gamma Y_{a} .
\end{aligned}
$$

When a single brand is adopted by all downstream firms (say brand $k$ ), we can use the above analysis to express the inverse demand for the intermediate good to be transformed in brand $k$ as $w_{k}=1-\frac{n+1}{n} Y_{k}$.

\subsection{Upstream firms' decisions}

Let us consider the case where the two brands have been selected by a positive number of downstream and upstream firms. Since upstream firms have the opportunity to produce the intermediate input for either brand, we need a slightly different notation. Let the upstream industry be partitioned into the two subsets $M_{a}$ and $M_{b}\left(M_{a} \cap M_{b}=\emptyset, M_{a} \cup M_{b}=M\right)$ according to the firms' brand choices. Let $x_{i k}$ denote the quantity of the intermediate good produced by the upstream firm $i(i=1, \ldots, m)$ to be transformed into brand $k(k=a, b)$, and let the total quantity, $X_{k}$, of the intermediate good to be transformed into brand $k$ be defined as

$$
X_{k} \equiv X_{a k}+X_{b k} \equiv \sum_{i \in M_{a}} x_{i k}+\sum_{j \in M_{b}} x_{j k} .
$$


Because of the linear technology in the downstream industry, we have the following market clearing conditions: $Y_{a}=X_{a}$ and $Y_{b}=X_{b}$. Using the latter conditions and expressions (3) and (4), we can write the upstream firms' maximization program for a typical upstream firm $i \in M_{a}$ as follows:

$$
\begin{aligned}
\max _{x_{i a}, x_{i b}} \Pi_{i}^{u}= & w_{a} x_{i a}+\left(w_{b}-t\right) x_{i b} \\
= & {\left[1-\frac{n+\Delta_{n}+2}{n+\Delta_{n}}\left(x_{i a}+X_{a}^{-i}\right)-\gamma\left(x_{i b}+X_{b}^{-i}\right)\right] x_{i a}+} \\
& {\left[1-t-\frac{n-\Delta_{n}+2}{n-\Delta_{n}}\left(x_{i b}+X_{b}^{-i}\right)-\gamma\left(x_{i a}+X_{a}^{-i}\right)\right] x_{i b} . }
\end{aligned}
$$

A similar expression obtains for the profit $\Pi_{j}^{u}$ of a typical firm $j \in M_{b}$.

Maximizing $\Pi_{i}^{u}$ with respect to $x_{i a}$ and $x_{i b}$, and $\Pi_{j}^{u}$ with respect to $x_{j a}$ and $x_{j b}$, we get a system of four first-order conditions that we solve using the symmetry of the model (at the symmetric equilibrium, we have $x_{i a}=x_{a a}$ and $x_{i b}=x_{a b}, \forall i \in M_{a}$, and $x_{j a}=x_{b a}$ and $x_{j b}=x_{b b}, \forall j \in M_{b}$ ). Accordingly, we get the following interior solutions (with $\Delta_{m} \equiv m_{a}-m_{b}$ ):

$$
\begin{aligned}
x_{a a}\left(\Delta_{n}, \Delta_{m}\right)= & \frac{\left(n+\Delta_{n}\right)\left(n-\Delta_{n}+2\right)\left[2+\left(m-\Delta_{m}\right) t\right]}{2(m+1)\left[4(n+1)+\left(1-\gamma^{2}\right)\left(n^{2}-\Delta_{n}^{2}\right)\right]} \\
& -\frac{\gamma\left(n^{2}-\Delta_{n}^{2}\right)\left[2-\left(m-\Delta_{m}+2\right) t\right]}{2(m+1)\left[4(n+1)+\left(1-\gamma^{2}\right)\left(n^{2}-\Delta_{n}^{2}\right)\right]}, \\
x_{a b}\left(\Delta_{n}, \Delta_{m}\right)= & \frac{\left(n-\Delta_{n}\right)\left(n+\Delta_{n}+2\right)\left[2-\left(m-\Delta_{m}+2\right) t\right]}{2(m+1)\left[4(n+1)+\left(1-\gamma^{2}\right)\left(n^{2}-\Delta_{n}^{2}\right)\right]} \\
& -\frac{\gamma\left(n^{2}-\Delta_{n}^{2}\right)\left[2+\left(m-\Delta_{m}\right) t\right]}{2(m+1)\left[4(n+1)+\left(1-\gamma^{2}\right)\left(n^{2}-\Delta_{n}^{2}\right)\right]}, \\
x_{b a}\left(\Delta_{n}, \Delta_{m}\right)= & x_{a b}\left(-\Delta_{n},-\Delta_{m}\right), \text { and } x_{b b}\left(\Delta_{n}, \Delta_{m}\right)=x_{a a}\left(-\Delta_{n},-\Delta_{m}\right) .
\end{aligned}
$$

This interior solution holds provided that $x_{a b}$ and $x_{b a}$ are both nonnegative, i.e., provided that $t$ is not too large. It is indeed clear that the upstream firms will only find it profitable to produce for the other brand than the one they have chosen provided that $t$ is low enough. Formally, $x_{a b} \geq$ $0 \Leftrightarrow t \leq \hat{t}_{a b}\left(\Delta_{n}, \Delta_{m}\right)$, and $x_{b a} \geq 0 \Leftrightarrow t \leq \hat{t}_{b a}\left(\Delta_{n}, \Delta_{m}\right)=\hat{t}_{a b}\left(-\Delta_{n},-\Delta_{m}\right)$. We have that $\hat{t}_{a b}$ decreases with $\Delta_{n}$ and increases with $\Delta_{m}$. We wish to focus on situations where the interior solution holds (i.e., where all upstream firms find it profitable to produce the intermediate good for the two brands) whatever the brand choices made in the two industries at the first stage of the game. A sufficient condition for this to be true is

$$
t \leq \frac{n(1-\gamma)+\gamma}{n+m[n(1+\gamma)-\gamma]}
$$


We assume that this condition is met throughout the rest of the paper which proves particularly useful for specification of equilibria in the first stage (we discuss the raison d'être of this assumption in Subsection 4.4).

\subsection{Equilibrium profits}

Because of the market clearing conditions and of the symmetry of the downstream firms, we have $y_{a}=\left(1 / n_{a}\right)\left(m_{a} x_{a a}+m_{b} x_{b a}\right)$ and $y_{b}=\left(1 / n_{b}\right)\left(m_{a} x_{a b}+\right.$ $\left.m_{b} x_{b b}\right)$. Plugging the latter expressions into (3) and (4), we get the equilibrium prices for the intermediate input to be transformed in the two brand:

$$
w_{a}\left(\Delta_{m}\right)=\frac{2+\left(m-\Delta_{m}\right) t}{2(m+1)} \text { and } w_{b}\left(\Delta_{m}\right)=w_{a}\left(-\Delta_{m}\right) .
$$

Not surprisingly, we observe that $w_{a} \gtreqless w_{b} \Leftrightarrow \Delta_{m} \lesseqgtr 0$, and that the difference $\left(w_{a}-w_{b}\right)$ decreases with $\Delta_{m}$. In other words, the more upstream firms select some brand, the cheaper the input to be transformed into that brand. Substituting the input prices into expressions (1) and (2), we obtain the equilibrium quantities for the downstream firms as:

$$
\begin{aligned}
y_{a}\left(\Delta_{n}, \Delta_{m}\right)= & \frac{\left(n-\Delta_{n}+2\right)\left[m(2-t)+\Delta_{m} t\right]}{2(m+1)\left[4(n+1)+\left(1-\gamma^{2}\right)\left(n^{2}-\Delta_{n}^{2}\right)\right]} \\
& -\frac{\gamma\left(n-\Delta_{n}\right)\left[m(2-t)-\Delta_{m} t\right]}{2(m+1)\left[4(n+1)+\left(1-\gamma^{2}\right)\left(n^{2}-\Delta_{n}^{2}\right)\right]}, \\
y_{b}\left(\Delta_{n}, \Delta_{m}\right)= & y_{a}\left(-\Delta_{n},-\Delta_{m}\right) .
\end{aligned}
$$

As above, $\Pi_{a}^{d}\left(\Delta_{n}, \Delta_{m}\right)=\left[y_{a}\left(\Delta_{n}, \Delta_{m}\right)\right]^{2}$ and $\Pi_{b}^{d}\left(\Delta_{n}, \Delta_{m}\right)=\left[y_{b}\left(\Delta_{n}, \Delta_{m}\right)\right]^{2}$. Using the previous results, we can also to derive the equilibrium profits in the upstream industry:

$$
\begin{aligned}
\Pi_{a}^{u}\left(\Delta_{n}, \Delta_{m}\right)= & \frac{\left(n+\Delta_{n}\right)\left(n-\Delta_{n}+2\right)\left[2-\left(m-\Delta_{m}\right) t\right]^{2}}{2(m+1)^{2}\left[4(n+1)+\left(1-\gamma^{2}\right)\left(n^{2}-\Delta_{n}^{2}\right)\right]} \\
& +\frac{\left(n-\Delta_{n}\right)\left(n+\Delta_{n}+2\right)\left[2-\left(m-\Delta_{m}+1\right) t\right]^{2}}{2(m+1)^{2}\left[4(n+1)+\left(1-\gamma^{2}\right)\left(n^{2}-\Delta_{n}^{2}\right)\right]} \\
& -\frac{\gamma\left(n^{2}-\Delta_{n}^{2}\right)\left[2-\left(m-\Delta_{m}\right) t\right]\left[2-\left(m-\Delta_{m}+1\right) t\right]}{2(m+1)^{2}\left[4(n+1)+\left(1-\gamma^{2}\right)\left(n^{2}-\Delta_{n}^{2}\right)\right]}, \\
\Pi_{b}^{u}\left(\Delta_{n}, \Delta_{m}\right)= & \Pi_{a}^{u}\left(-\Delta_{n},-\Delta_{m}\right)
\end{aligned}
$$

As far as Condition (INT) holds, the previous expressions are easily used to describe limit cases where all firms in some industry select the same 
brand. We have thus all necessary elements at our disposal to analyze the first-stage brand choices in the two industries.

Before considering the general framework in depth, let us quickly relate it to the two special cases analyzed above. First, if we set $t=0$ in the general setting, we come back to the first special case where $w_{a}=w_{b}$. Second, exogenous Marshallian externalities can be obtained in the general setting by making the following assumptions: (i) $m=x n$, where $x$ is an integer larger than or equal to 1, (ii) each downstream firm can dictate its choice of brand to a separate set of $x$ upstream firms. These assumptions imply that $\Delta_{m}=x \Delta_{n}$. As a result, some manipulations allow to rewrite $w_{a}\left(\Delta_{m}\right)$ as $w-\theta n_{a}$, with $w=(1+x n t) /(x n+1)$ and $\theta=(x t) /(x n+1)$.

In these two special cases, downstream firms are either splitting equally or concentrating on a single brand. We want now to investigate whether the set of equilibria expands when upstream firms are free to choose the brand they want to produce for, and when the adaptation cost $t$ is positive (although small enough for Condition (INT) to hold). The answer to this question will be shown to depend on the balance between the following two conflicting forces. On the one hand, downstream firms have an incentive to choose different brands in order to soften competition on the final market; stability then requires that they split equally between the two brands. On the other hand, brand choices also determine the relative price of the input; this second force drives downstream firms to model their behavior on that of the upstream firms. Since upstream firms tend to do exactly the same, this force drives the market towards unanimous adoption of a single brand.

In what follows, we look for Nash equilibria in the brand choice game, i.e., for partitions of the two sets of firms such that no firm-either upstream or downstream-has an incentive to unilaterally switch brands. We establish the existence of a Nash equilibrium and draw instructive results about the characterization of the set of Nash equilibria.

\subsection{Existence and characterization of equilibria}

The brand choice game might lead to two typical industry patterns: either no industry is concentrated on a single brand (a pattern referred to hereafter as dispersion), or all firms in at least one industry decide to adopt the same brand (a pattern referred to hereafter as concentration, with complete concentration denoting the situation where firms in both industries adopt 
the same brand).

When there is dispersion $\left(\left|\Delta_{n}\right|<n\right.$ and $\left|\Delta_{m}\right|<m$ ), four conditions have to be met to guarantee a Nash equilibrium: no downstream firm finds it profitable to deviate if and only if (Da) $\Pi_{a}^{d}\left(\Delta_{n}, \Delta_{m}\right) \geq \Pi_{b}^{d}\left(\Delta_{n}-2, \Delta_{m}\right)$, and (Db) $\Pi_{b}^{d}\left(\Delta_{n}, \Delta_{m}\right) \geq \Pi_{a}^{d}\left(\Delta_{n}+2, \Delta_{m}\right)$; similarly, deviation by upstream firms is unprofitable if and only if (Ua) $\Pi_{a}^{u}\left(\Delta_{n}, \Delta_{m}\right) \geq \Pi_{b}^{u}\left(\Delta_{n}, \Delta_{m}-2\right.$ ), and (Ub) $\Pi_{b}^{u}\left(\Delta_{n}, \Delta_{m}\right) \geq \Pi_{a}^{u}\left(\Delta_{n}, \Delta_{m}+2\right)$.

Using the expressions of second-stage equilibrium profits, we can develop the latter four conditions as follows (with $t>0$ ):

$$
\begin{aligned}
& \text { (Da) } \Delta_{m} \geq \frac{m(2-t)(1-\gamma)}{t} \Phi\left(\Delta_{n}\right), \\
& \text { (Db) } \Delta_{m} \leq \frac{m(2-t)(1-\gamma)}{t} \Phi\left(\Delta_{n}+2\right), \\
& \text { (Ua) } \Delta_{m} \leq \frac{2(2-t)}{t} \Psi\left(\Delta_{n}\right)+1, \\
& \text { (Ub) } \Delta_{m} \geq \frac{2(2-t)}{t} \Psi\left(\Delta_{n}\right)-1,
\end{aligned}
$$

where

$$
\begin{aligned}
\Phi\left(\Delta_{n}\right) & =\frac{\left(\Delta_{n}-1\right)\left[[n(1-\gamma)+2][n(1+\gamma)-2 \gamma]-\Delta_{n}\left(\Delta_{n}-2\right)\left(1-\gamma^{2}\right)\right]}{[n(1+\gamma)+2]\left[\left(1-\gamma^{2}\right) n^{2}+\left(3+\gamma^{2}\right) n-2 \gamma(1-\gamma)\right]-\Delta_{n}\left(\Delta_{n}-2\right)\left(1-\gamma^{2}\right)[3+\gamma+n(1+\gamma)]}, \\
\Psi\left(\Delta_{n}\right) & =\frac{\Delta_{n}}{2 n+\left(n^{2}-\Delta_{n}^{2}\right)(1+\gamma)} .
\end{aligned}
$$

The same set of conditions applies to analyze situations of concentration, except that one or the other condition will become irrelevant. ${ }^{12}$

To establish the existence of an equilibrium and characterize the set of possible equilibria, we need to examine closely the two functions $\Psi\left(\Delta_{n}\right)$ and $\Phi\left(\Delta_{n}\right)$. The next lemma summarizes their useful properties. The proofs of this lemma and of all subsequent propositions are relegated to Section 7 .

Lemma 1 (i) For all $-n \leq \Delta_{n} \leq n, \Psi\left(\Delta_{n}\right)=-\Psi\left(-\Delta_{n}\right)$.

(ii) For all $-n \leq \Delta_{n} \leq n-2, \Phi\left(\Delta_{n}+2\right)=-\Phi\left(-\Delta_{n}\right)$.

(iii) For all $-n \leq \Delta_{n} \leq n-2, \Psi\left(\Delta_{n}+2\right)>\Psi\left(\Delta_{n}\right)$.

(iv) $\Phi\left(\Delta_{n}\right) \geq 0$ has the sign of $\left(\Delta_{n}-1\right)$.

(v) For all $-n+2 \leq \Delta_{n} \leq n-2, \Phi\left(\Delta_{n}+2\right)>\Phi\left(\Delta_{n}\right)$.

\footnotetext{
${ }^{12}$ For instance, for the situation of global concentration on brand $a$ to be an equilibrium, only conditions (Ua) and (Da), with $\Delta_{m}=m$ and $\Delta_{n}=n$, have to be met.
} 
The first two statements are a direct consequence of the symmetry of the model. They imply that if conditions (Ua), (Ub), (Da), and (Db) are met for $\Delta_{m}=x$ and $\Delta_{n}=y$, they are also met for $\Delta_{m}=-x$ and $\Delta_{n}=$ $-y$. Next, we learn from statement (iv) that for $\Delta_{n}>0$ (resp. $<0$ ), conditions ( $\mathrm{Da})$ and $(\mathrm{Db})$ require that $\Delta_{m}$ be comprised between two nonnegative (resp. non-positive) bounds; this implies that it is impossible to have a brand choice equilibrium where some particular brand is chosen by a majority of downstream firms and a minority of upstream firms (or vice versa). Moreover, statements (iii) and (v) imply that in equilibrium, larger values of $\Delta_{m}$ necessarily correspond to larger values of $\Delta_{n}$ and vice versa. Finally, $\Phi(0)=-\Phi(2)<0$ means that conditions (Da) and (Db) impose no restriction on the sign of $\Delta_{m}$ in that particular case; however, using conditions (Ua) and (Ub), we see that if downstream firms split equally between the two brands $\left(\Delta_{n}=0\right)$, the only possible equilibrium is where upstream firms also split equally $\left(\Delta_{m}=0\right)$ between the two brands.

We have thus established two important characterization results about possible equilibria in the brand choice game. They are summarized in the following proposition.

Proposition 2 (i) Candidate equilibria are located in the North-East quadrant of the $\left(\Delta_{m}, \Delta_{n}\right)$ grid (with mirror-equilibria in the South-West quadrant). (ii) If equilibria coexist, they can be ranked in the sense that larger values of $\Delta_{n}$ correspond to larger values of $\Delta_{m}$.

Building on Lemma 1 and Proposition 2, we can now prove existence of an equilibrium in the brand choice game, as stated in Proposition 3.

Proposition 3 For all parameter values satisfying Condition (INT), there exists at least one Nash equilibrium in the first-stage brand choice game.

The proof of Proposition exploits the following two findings: (i) for every $\Delta_{m}$, there is a unique value of $\Delta_{n}$ such that no downstream firm finds it profitable to deviate, and (ii) the so-defined function of $\Delta_{m}$ is non-decreasing. The rest of the proof consists in showing that the latter function admits at least one fixed point.

Now that we have established the existence of equilibrium and excluded a whole category of industry partitions as possible equilibria (namely the North-West and South-East quadrants of the $\left(\Delta_{m}, \Delta_{n}\right)$ grid), we would 
like to better characterize equilibria according to the values of the two key parameters $t$ and $\gamma$. This task is relatively easy in two special cases, namely concentration and equal split of one or the other industry. The next two propositions record our results in these two cases.

Proposition 4 Suppose Condition (INT) is met. (i) Global concentration is a brand choice equilibrium provided that, for a given degree of product differentiation $\gamma$, the adaptation cost $t$ is large enough. More precisely, we need $t \geq \bar{t}(\gamma, n) \equiv[(n-1)(n-\gamma)(1-\gamma)] /[n(n+1)]$. (ii) If the downstream industry is concentrated, the only possible equilibrium is where the upstream industry is concentrated as well. The reverse is not true.

Note that $\bar{t}(\gamma, n)$ is a decreasing function of $\gamma$, meaning that the more brands are differentiated (or the lower $\gamma$ ) the narrower the range of adaptation costs $(t)$ supporting global concentration as an equilibrium. The intuition for this result is as follows. When brand differentiation increases, downstream firms have a higher incentive to choose different brands in order to soften competition on the final market. The counterweight lies in the higher input cost that would result from such a move and it clearly increases with $t$. It can also be checked that $\bar{t}(\gamma, n)$ is an increasing function of $n$ (and the intuition is similar to the previous one). Finally, noteworthy is the fact that $\bar{t}(\gamma, n)$ does not depend on $m$, the size of the upstream industry.

Proposition 5 Suppose Condition (INT) is met. When both industries count an even number of firms, equal splitting $\left(\Delta_{m}=\Delta_{n}=0\right)$ is a brand choice equilibrium for all admissible values of $t$ and $\gamma$. There is no other equilibrium with either $\Delta_{m}=0$ or $\Delta_{n}=0$.

Proposition 5 tells us that when both industries count an even number of firms, equal split in one industry calls for equal split in the other industry as well.

Unfortunately, except for the two previous cases, the complex form of the four conditions for equilibrium makes it impossible to explicitly characterize the set of equilibria according to the parameter values. However, we know from the above two propositions that multiple equilibria might occur (with $m$ and $n$ even, equal split is an equilibrium for all $t$ and $\gamma$, and global concentration is an equilibrium for $t \geq \bar{t}(n, \gamma))$. Moreover, numerical simulations allow us to ascertain the possibility of asymmetric equilibria. 
Take, for instance, $m=n=10, \gamma=0.95$, and $t=0.007$. We have then an equilibrium with $\Delta_{m}=6$ and $\Delta_{n}=2$. The next proposition records this important result.

Proposition 6 There exist constellations of parameters for which the brand choice equilibrium involves an unequal dispersion of firms in both industries.

\subsection{Discussion}

We briefly discuss here the raison d'être of Condition (INT), as well as the case of an odd number of firms in one or the other industry.

The raison d'être of Condition (INT) is best understood by considering what happens when it does not hold for all $\gamma$ and $t$. In such a case, the upstream firms that have selected brand $a$ (resp. brand $b$ ) can produce for brand $a$ and brand $b$, or for brand $a$ only (resp. brand $b$ only). There are thus four possible scenarios according to whether firms of each type produce either for both brands or for a single one. In contrast, if Condition (INT) holds, both types of upstream firms produce for both brands. As we have seen, the method used to check if $\left(\Delta_{n}, \Delta_{m}\right)$ is an equilibrium consists of comparing the profits made by each upstream firm at $\left(\Delta_{n}, \Delta_{m}\right)$ to the profits made at $\left(\Delta_{n}, \Delta_{m}-2\right)$ (when one upstream that had selected brand $a$ switches to brand $b$ ) and to the profits made at $\left(\Delta_{n}, \Delta_{m}+2\right)$ (when one upstream that had selected brand $b$ switches to brand $a$ ). On the one hand, when Condition (INT) does not hold for all $\gamma$ and $t$, there are four possible expressions for the profits at $\left(\Delta_{n}, \Delta_{m}\right)$, four other at $\left(\Delta_{n}, \Delta_{m}-2\right)$ and again four at $\left(\Delta_{n}, \Delta_{m}+2\right)$. There are thus 64 comparisons to make. On the other hand, when Condition (INT) holds, only two comparisons remain. As demonstrated above, the analysis is still analytically difficult to handle even with only two comparisons. This provides a practical vindication for our assumption. Referring to the motivation given in Section 1, we could also say that the FMS used in the upstream industry guarantees that $t$ be kept low enough whatever the market conditions.

Because brand choices by individual firms have a discrete impact in our model, it is not surprising that slightly different results obtain when one or the other industry counts an odd number of firms. In particular, it can be shown that if at least one industry counts an odd number of firms, 'almost' equal splitting $\left(\Delta_{m}, \Delta_{n} \in\{0,1\}\right)$ might not be an equilibrium for some values of $\gamma$ and $t$. (Yet, it can also be shown that this becomes less likely 
as the number of firms in the "odd" industry increases). The reason is that, because of the link between the two industries, the slight imbalance of the partition in the "odd" industry might make equal split unstable in the "even" industry. Fortunately, our results about the existence of an equilibrium, the characterization of equilibria with concentration, and the possibility of asymmetric equilibria hold independently of whether industries count an even or an odd number of firms.

\section{$5 \quad$ Welfare considerations}

We define total welfare as the sum of three elements: (i) total profits in the upstream industry, (ii) total profits in the downstream industry, and (iii) the consumer surplus. Before stating our results for the general setting, let us draw some insights from our two special cases with exogenous costs. When costs are constant and identical, it can be shown that private and social incentives coincide everywhere: equal split is the unique equilibrium and it maximizes total welfare. Noteworthy is the fact that while the consumer unambiguously prefers equal split over any other partition (because it provides her with the variety she loves at the relatively low cost of a reduced but balanced competition), the downstream industry as a whole might prefer some unequal partition (e.g., for $n=6$ and $\left.\gamma=0, \Pi^{d}(2)>\Pi^{d}(0)\right)$. The addition of an agglomeration force (Marshallian externalities) is a source of possible divergence between private and social incentives. It is also a source of complexity in the welfare analysis. One important result emerges, however, from the second special case: equal split leads to a higher total welfare than concentration provided that $\theta$ be lower than some threshold $\hat{\theta}$, which is itself smaller than $\bar{\theta}$ (i.e., the value of $\theta$ under which equal split is an equilibrium). This means that the market might yield excessive dispersion from a social point of view. ${ }^{13}$

When downstream costs are endogenized through the upstream choices, additional sources of market inefficiency appear. Not surprisingly, the same difficulties that we encounter for the characterization of the set of equilibria impede any exhaustive welfare analysis. In particular, taking the point of view of a second-best regulator, we are unable to determine the partition of the two industries that maximizes total welfare for a given set of parameters.

\footnotetext{
${ }^{13}$ The proofs for the statement can be obtained from the authors upon request.
} 
The difficulty (revealed by numerical simulations) comes from the fact that total welfare might well reach its highest value for asymmetric partitions of the two industries (be they equilibria or not). Nevertheless, some instructive results can be drawn from the comparison of global concentration and equal split, where expressions are easier to handle. Such comparison makes perfect sense when $m$ and $n$ are even. Indeed, we know that equal split and global concentration will be equilibria simultaneously for $t \geq \bar{t}(n, \gamma)$. We might therefore wonder whether the different agents and society as a whole have a clear preference for one or the other equilibrium. The next proposition records our results.

Proposition 7 Suppose $m$ and $n$ even. Call $S$ the set of parameters where global concentration and equal split are equilibria simultaneously. The set of parameters where the representative consumer prefers global concentration to equal split includes $S$, whereas the set of parameters where (downstream and upstream) firms prefer global concentration to equal split is included in S. Ambiguous results obtain for society as a whole.

The intuition for Proposition 7 can be explained as follows. For the representative consumer, the advantage of equal split is brand variety, but the disadvantage is higher prices because of higher input costs. In other words, the consumer will prefer total concentration to equal split provided that $t$ be large enough for a given $\gamma$. It turns out that an even larger value of $t$ is required for the firms not to deviate from total concentration. Therefore, when total concentration is an equilibrium, the consumer unambiguously prefers it to equal split. Regarding downstream firms, equal split leads to softer competition but higher input costs than total concentration. However, for a given $\gamma$, the lowest value of $t$ that induces the downstream industry, as a whole, to favor total concentration is larger than the one that prevents individual deviations from total concentration. Accordingly, total concentration being an equilibrium does not imply that the downstream industry prefers it to equal split. The same conclusion applies for the upstream industry, yet for other reasons. On the one hand, we have shown that upstream firms do not wish to deviate from total concentration as long as the downstream industry is concentrated. On the other hand, the demand for the final goodand hence for the input-decreases with $\gamma$ which explains why upstream firms favor equal split unless both $t$ and $\gamma$ are high enough. It turns out that the set of parameters where the latter is true is included in $S$. 
In sum, the interests of the consumer are at odds with those of the firms, which justifies that there is no clear cut conclusion for society as a whole: when total concentration and equal split are both equilibria, the ranking of their welfare performance depends on the values of the parameters. In other words, when total concentration is an option, the second-best regulator does not necessarily prefer it to equal split.

\section{Conclusion}

In this paper, we have examined the softening effect of product differentiation on price competition in a model of successive oligopolies with two brands. Downstream firms select the brand that they sell on a final market. Each upstream firm specializes in the production of one input specifically designed for the production of one brand in the downstream industry. They incur an extra cost when they produce for the other brand.

We have shown that the larger is the number of downstream firms that select one brand, the larger is the number of upstream firms that specialize in the input specific to that brand, and vice versa. Hence, downstream firms faces the following trade-off. By selecting the brand that is the less popular among downstream firms, they soften the competition and therefore increase their profits. This is the effect that is emphasized in the economics and marketing literature. However, this choice is also associated with a low pool of suppliers and high input costs, which decreases profits. This effect is similar to the 'cost linkage' identified in the new economic geography literature (see e.g. Venables (1996)). This paper shares this effect with that literature but examines the location of firms in a product rather than in a geographical space.

We have proved that there exists always at least one equilibrium in the brand choice game. Multiple equilibria might also occur. We have examined in details two prominent equilibrium candidates, namely concentration and equal split. In the first case, downstream and upstream firms are unanimous to choose a single brand: there is no product differentiation. This is an equilibrium provided that the extra cost of adapting one input designed for one brand to the other brand is large enough. In the second case, half (or half plus or minus one) downstream and upstream firms choose one brand, the other half choose the other brand. This maximal differentiation situation is 
always an equilibrium when there is an even number of firms in each industry. We have also shown that other equilibria without maximal differentiation might also exist. Finally, we have examined the welfare performance of these equilibria. This is unfortunately not an easy task and there is no clear-cut conclusion to draw as a whole. However, we have established that the interests of the consumer are at odds with those of the firms.

The model developed in this paper has been kept as simple as possible in order to highlight the interplay between the brand choices in the two vertically linked industries. Despite the relative simplicity of our two stagegame, we have not been able to fully characterize the set of its equilibria, nor to gauge their welfare performance. More than an acknowledgment of partial failure, we have here a clear demonstration of the complexity and significance of the strategic interaction addressed in the model. We believe this should fuel further research on the topic, and we suggest therefore some extensions of the model. It might indeed be the case that some more insights would be gained in a slightly richer model.

A first extension would be to endogenize the degree of product differentiation between the two brands of the final product. We could assume that the downstream firms have the opportunity to invest in some technology that allows them to increase product differentiation. As in Lambertini and Rossini (1998) or in Belleflamme (2000), we would then express $\gamma$ as a decreasing function of the number of firms investing in this technology; it seems indeed natural to suppose that brands become more differentiated as more firms invest in differentiation.

A second extension would be to make the adaptation cost $t$ depend on the degree of product differentiation $\gamma$. It seems indeed more natural to assume that this adaptation cost becomes larger the more the brands of the final product are differentiated.

The last two directions for further research seem more far-reaching. On the one hand, it might be worth investigating the possibility of vertical arrangements between downstream and upstream firms (e.g., vertical integration, formation of 'production networks', or more generally, all forms of "supply chain management", as it is called in the business literature). On the other hand, we could let downstream firms produce the two differentiated brands if they wish to (in other words, downstream firms would have access to the same type of flexible manufacturing systems as upstream firms 
are supposed to use). The decision to produce another brand (or other brands) in addition to the initial one can be seen as a movement towards mass customisation (i.e., the provision of such a variety of products that nearly everyone can find what they want). But customisation is likely to increase production costs in a similar way as in our model. As Alford et al. (2000) report about the automotive industry, "The desire to offer customers numerous choices of appearance, performance, comfort, safety and security is at the expense of escalating complexity and cost in assembly and the supply chain." 14

\section{Appendix}

Proof of Lemma 1. The proofs of statements (i), (ii), and (iii) are straightforward. Statements (iv) and (v) are less obvious. Let us adopt the following notation. Let $A \equiv[n(1-\gamma)+2], B \equiv[n(1+\gamma)-2 \gamma], C \equiv$ $\left(1-\gamma^{2}\right), D \equiv[n(1+\gamma)+2], E \equiv\left[\left(1-\gamma^{2}\right) n^{2}+\left(3+\gamma^{2}\right) n-2 \gamma(1-\gamma)\right]$, and $F \equiv[3+\gamma+n(1+\gamma)]$, which are all strictly positive for $\gamma<1$.

We have thus that $\Phi\left(\Delta_{n}\right) \geq 0$ if and only if

$$
\frac{\left(\Delta_{n}-1\right)\left[A B-C \Delta_{n}\left(\Delta_{n}-2\right)\right]}{D E-C F \Delta_{n}\left(\Delta_{n}-2\right)}>0 .
$$

The two terms $\left[A B-C \Delta_{n}\left(\Delta_{n}-2\right)\right]$ and $\left[D E-C F \Delta_{n}\left(\Delta_{n}-2\right)\right]$ define parabolas in $\Delta_{n}$ with a maximum at $\Delta_{n}=0$ (1) if $n$ is even (odd). It is thus easily seen that both terms reach their lowest possible value at $\Delta_{n}=n$ or $\Delta_{n}=-n+2$, i.e., respectively, $4(n-\gamma)$ and $4(1+\gamma) n^{2}+4\left(3-\gamma^{2}\right) n-4 \gamma(1-\gamma)$. The former term is clearly strictly positive; the latter term increases with $n$ and reaches thus is lowest value for $n=2$, which is $4[10+\gamma(3-\gamma)]>0$. It follows that $\Phi\left(\Delta_{n}\right)$ has the sign of $\left(\Delta_{n}-1\right)$ which proves statement (iv).

Regarding statement (v), we have that $\Phi\left(\Delta_{n}+2\right)>\Phi\left(\Delta_{n}\right)$ if and only

\footnotetext{
${ }^{14}$ Similarly, Fisher (1997) quotes the experience of the Japanese firm National Bicycle to illustrate that mass customization is not necessarily cheap: "National's custom production requires three times more labor than assembly-line production of bikes."
} 


$$
\begin{aligned}
& \frac{\left(\Delta_{n}+1\right)\left[A B-C \Delta_{n}\left(\Delta_{n}+2\right)\right]}{D E-C F \Delta_{n}\left(\Delta_{n}+2\right)}>\frac{\left(\Delta_{n}-1\right)\left[A B-C \Delta_{n}\left(\Delta_{n}-2\right)\right]}{D E-C F \Delta_{n}\left(\Delta_{n}-2\right)} \\
\Leftrightarrow & \frac{2\left[C^{2} F \Delta_{n}^{4}+C(A B F-3 D E-4 C F) \Delta_{n}^{2}+A B D E\right]}{\left[D E-C F \Delta_{n}\left(\Delta_{n}+2\right)\right]\left[D E-C F \Delta_{n}\left(\Delta_{n}-2\right)\right]}>0 \\
\Leftrightarrow & \frac{2\left(C \Delta_{n}^{2}-A D\right)\left(C F \Delta_{n}^{2}-B E\right)}{\left[D E-C F \Delta_{n}\left(\Delta_{n}+2\right)\right]\left[D E-C F \Delta_{n}\left(\Delta_{n}-2\right)\right]}>0,
\end{aligned}
$$

the last step making use of the definitions of $A$ to $F$. Because $C \Delta_{n}^{2}-A D=$ $-\left[\left(1-\gamma^{2}\right)\left(n^{2}-\Delta_{n}^{2}\right)+4(n+1)\right]<0$, we have that $\Phi\left(\Delta_{n}+2\right)>\Phi\left(\Delta_{n}\right) \Leftrightarrow$ $\left(\Delta_{n}\right)>0$, where

$$
\left(\Delta_{n}\right)=\frac{B E-C F \Delta_{n}^{2}}{\left[D E-C F \Delta_{n}\left(\Delta_{n}+2\right)\right]\left[D E-C F \Delta_{n}\left(\Delta_{n}-2\right)\right]} .
$$

Since $\left(\Delta_{n}\right)=\left(-\Delta_{n}\right)$, we can focus, without loss of generality on $\Delta_{n} \geq 0$. Now, it is easy to see the following. (1) For $\Delta_{n} \geq 0, D E-C F \Delta_{n}\left(\Delta_{n}-2\right)>$ $D E-C F \Delta_{n}\left(\Delta_{n}+2\right)$. (2) The term $D E-C F \Delta_{n}\left(\Delta_{n}+2\right)$ reaches its lowest value for $\Delta_{n}=n-2$, which is equal to $4(1+\gamma) n^{2}+4\left(3-\gamma^{2}\right) n-4 \gamma(1-\gamma)>0$ for all $\gamma \in[0,1]$ and $n \geq 2$. (3) The term $B E-C F \Delta_{n}^{2}$ also reaches its lowest value for $\Delta_{n}=n-2$, which is equal to $4(1+\gamma) n^{2}+8\left(1-\gamma-\gamma^{2}\right) n-4(3+\gamma+$ $\left.4 \gamma^{2}\right)$. It is easy to show that this polynomial in $n$ admits two real roots; for all $\gamma \in[0,1]$, the first root is negative, and the second is positive but strictly inferior to 2 , meaning that the polynomial is strictly positive for all $\gamma \in[0,1]$ and $n \geq 2$. We have thus proved that $\left(\Delta_{n}\right)>0 \forall 0 \leq \Delta_{n} \leq n-2$. Since $\left(\Delta_{n}\right)=\left(-\Delta_{n}\right)$, this also means that $\left(\Delta_{n}\right)>0 \forall-n+2 \leq \Delta_{n} \leq 0$, which completes the proof.

Proof of Proposition 3. The first step of the proof consists in showing that for every $\Delta_{m}$, there is a unique value of $\Delta_{n}$ such that no downstream firm finds it profitable to deviate. Conditions ( $\mathrm{Da}$ ) and (Db), combined with statement (v) of Lemma 1, imply indeed that by varying $\Delta_{n}$ from $-n$ to $n$ and imposing equilibrium in the downstream industry, we define an increasing sequence of adjacent non-empty intervals which completely covers the real line. (The first interval is given by $[-\infty,(m(2-t)(1-\gamma)) \Phi(-n+2) / t]$, and the last one by $[(m(2-t)(1-\gamma)) \Phi(n) / t, \infty])$. This means that any value of $\Delta_{m}$ falls in one and only one of these intervals. We can thus define the non-decreasing function $D\left(\Delta_{m}\right)$ that associates to every $\Delta_{m}$ a unique value of $\Delta_{n}$ for which there is equilibrium in the downstream industry. 
Take now $\Delta_{m}=x_{0}$, with $x_{0}<m$, and let $y_{0}=D\left(x_{0}\right)$. If the pair $\left(x_{0}, y_{0}\right)$ also meets conditions ( $\mathrm{Ua})$ and $(\mathrm{Ub})$, we are done. Otherwise, we have that either (Ua) or (Ub) is violated. Suppose without loss of generality that (Ub) is violated, meaning that for $\Delta_{n}=y_{0}$, upstream firms wish to switch from brand $a$ to brand $b$. Consider then $x_{1}=x_{0}+2$. We know that $y_{1}=D\left(x_{1}\right) \geq$ $y_{0}$. Again, if the pair $\left(x_{1}, y_{1}\right)$ meets $(\mathrm{Ua})$ and $(\mathrm{Ub})$, we are done. Otherwise, we have necessarily that (Ub) is violated. To see this, remember that (Ub) was violated for $\left(x_{0}, y_{0}\right)$; that is, we had $x_{0}<[2(2-t) / t] \Psi\left(y_{0}\right)-1$, which is equivalent to $x_{1}=x_{0}+2<[2(2-t) / t] \Psi\left(y_{0}\right)+1 \leq[2(2-t) / t] \Psi\left(y_{1}\right)+1$ (since $y_{1} \geq y_{0}$ and $\Psi\left(\Delta_{n}\right)$ is an increasing function of $\Delta_{n}$ ), which ensures that (Ua) is met for $\left(x_{1}, y_{1}\right)$. In other words, upstream firms still wish to switch from brand $a$ to brand $b$. We can thus repeat the previous procedure. In the worst-case scenario, this will drive us to $x_{k}=m$, with a corresponding $y_{k}=D(m) \geq y_{k-1}=D(m-2)$. For $x_{k}=m$, all upstream firms adopt brand $a$. Therefore, equilibrium in the upstream industry only requires that condition (Ua) be satisfied. Using our previous argument, we know that this is necessarily true since condition (Ub) was violated for $\left(x_{k-1}, y_{k-1}\right)$.

A analog argument applies if, starting from the pair $\left(x_{0}, y_{0}\right)$, we assume instead that condition (Ua) is not met.

Proof of Proposition 4. (i) For global concentration to be a brand choice equilibrium, only conditions ( $\mathrm{Ua}$ ) and (Da) have to be met. With $\Delta_{m}=m$ and $\Delta_{n}=n$, these two conditions translate as:

$$
\frac{m(2-t)(1-\gamma)}{t} \quad \Phi(n) \leq m \leq \frac{2(2-t)}{t} \quad \Psi(n)+1 .
$$

It is easy to see that second inequality (which ensures no deviation in the upstream industry) boils down to $m \leq 2 / t$, which is clearly satisfied because of Condition (INT). On the other hand, developing the first inequality, we have

$$
\begin{aligned}
m & \geq \frac{m(2-t)(1-\gamma)}{t} \frac{4(n-1)(n-\gamma)}{4(1+\gamma) n^{2}+4\left(3-\gamma^{2}\right) n-4 \gamma(1-\gamma)} \Leftrightarrow \\
t & \geq \frac{(n-1)(n-\gamma)(1-\gamma)}{n(n+1)} \equiv \bar{t}(\gamma, n) .
\end{aligned}
$$

(ii) Let $\Delta_{n}=n$ and $\Delta_{m}=x<m$. For this to be an equilibrium, condition (Ub) has to be met, i.e., $x \geq \Psi(n)[2(2-t)] / t-1$, which is equivalent to $x+2 \geq \Psi(n)[2(2-t)] / t+1=2 / t$. This is impossible since we have 
established above that $m \leq 2 / t$. We have thus proved that there is no equilibrium with $\Delta_{n}=n$ and $\Delta_{m}=m$. However, equilibria with $\Delta_{m}=m$ and $\Delta_{n}<n$ are possible. For instance, with $m=n=3, \Delta_{m}=3$ and $\Delta_{n}=1$ is an equilibrium for $\gamma=0.5$ and $t=0.1$.

Proof of Proposition 5. Take $\Delta_{n}=0$. Conditions (Ua) and (Ub) can be rewritten as $-1 \leq \Delta_{m} \leq 1$, while conditions ( $\mathrm{Da}$ ) and ( $\mathrm{Db}$ ) boil down to $[m(2-t)(1-\gamma)] \Phi(0) \leq t \Delta_{m} \leq[m(2-t)(1-\gamma)] \Phi(2)$. With $m$ even, both are true only for $\Delta_{m}=0$ since we know from statement (iv) of Lemma 1 that $\Phi(0)=-\Phi(2)<0$. Take now $\Delta_{m}=0$. We know from Proposition 2 that equilibria are only possible for $\Delta_{n} \geq 0$. We also know from statement (iv) of Lemma 1 that, with $n$ even, $\Phi\left(\Delta_{n}\right)>0$ for $\Delta_{n}>0$. Hence, condition (Da) can only be met for $\Delta_{n}=0$.

Proof of Proposition 7. With $m$ and $n$ even, the set $S$ of parameters where global concentration and equal split are equilibria simultaneously is defined by $\{m \geq 2, n \geq 2, t<1,0 \leq \gamma \leq 1 \mid t \geq \bar{t}(n, \gamma)=$ $[(n-1)(n-\gamma)(1-\gamma)] /[n(n+1)]\}$.

(1) Consumer. The demand functions for brands $a$ and $b$ are generated by a representative consumer who has the following quadratic utility function:

$$
U\left(Y_{a}, Y_{b}\right)=Y_{a}+Y_{b}-(1 / 2)\left(Y_{a}^{2}+Y_{b}^{2}\right)-\gamma Y_{a} Y_{b}+Y_{0}
$$

where $Y_{k}(k=a, b)$ is the quantity of brand $k$ and $Y_{0}$ the quantity of numéraire she consumes. We have $0 \leq \gamma \leq 1$. Her budget constraint is $R=p_{a} Y_{a}+p_{b} Y_{b}+Y_{0}$. Maximizing (7) subject to the budget constraint yields the linear inverse demand schedule presented in Section 2. Consumer surplus is equal to $C S \equiv U\left(Y_{a}, Y_{b}\right)-R$, i.e., using the inverse demand functions,

$$
C S=\frac{1}{2}\left(Y_{a}^{2}+Y_{b}^{2}\right)+\gamma Y_{a} Y_{b}
$$

$>$ From expressions (5) and (6), it is easy to compute the consumer surplus for the cases of global concentration and equal split, respectively as

$$
\begin{aligned}
C S(m, n) & =\frac{m^{2} n^{2}}{2(m+1)^{2}(n+1)^{2}}, \\
C S(m / 2, n / 2) & =\frac{m^{2} n^{2}(1+\gamma)(2-t)^{2}}{4(m+1)^{2}[n(1+\gamma)+2]^{2}} .
\end{aligned}
$$


One computes $C S(m, n)-C S(m / 2, n / 2)$. This is a quadratic form in $t$ with two real roots, one of which being larger than 1 . Therefore $C S(m, n)>$ $C S(m / 2, n / 2)$ if and only if

$$
t>t^{c}(n, \gamma) \equiv 2-\frac{[n(1+\gamma)+2] \sqrt{2(1+\gamma)}}{(n+1)(1+\gamma)} .
$$

Some lines of easy but tedious algebra establish that $t^{c}(n, \gamma)<\bar{t}(n, \gamma), \forall n \geq$ $2, \gamma \in[0,1]$, which proves that the set of parameters where the representative consumer prefers global concentration to equal split includes $S$.

(2) Downstream firms. Using the equilibrium profits derived in Subsection 4.2 , one derives the downstream industry total profits for the cases of global concentration and equal split, respectively as

$$
\begin{aligned}
\Pi^{d}(m, n) & =\frac{m^{2} n}{(m+1)^{2}(n+1)^{2}} . \\
\Pi^{d}(m / 2, n / 2) & =\frac{m^{2} n(2-t)^{2}}{(m+1)^{2}[n(1+\gamma)+2]^{2}} .
\end{aligned}
$$

It is straightforward to check that $\Pi^{d}(m, n)>\Pi^{d}(m / 2, n / 2)$ if and only if

$$
t>t^{d}(n, \gamma) \equiv \frac{n(1-\gamma)}{n+1}>\bar{t}(n, \gamma)
$$

which proves that the set of parameters where downstream firms prefer global concentration to equal split is included in $S$.

(3) Upstream firms. Using the equilibrium profits derived in Subsection 4.2 , one derives the upstream industry total profits for the cases of global concentration and equal split, respectively as

$$
\begin{aligned}
\Pi^{u}(m, n) & =\frac{m n}{(m+1)^{2}(n+1)}, \\
\Pi^{u}(m / 2, n / 2) & =\frac{m n\left\{[m(m+2)[n(1+\gamma)+2]+2(n+2)] t^{2}+4(1-t)[n(1-\gamma)+2]\right\}}{(m+1)^{2}[n(1+\gamma)+2][n(1-\gamma)+2]} .
\end{aligned}
$$

Computing $\Pi^{u}(m, n)-\Pi^{u}(m / 2, n / 2)$, we get a quadratic form in $t$. It can be shown that this quadratic form has no real root and is negative for $m(m+2)<2 /[n(1-\gamma)]$ (which, given that $m \geq 2$ and $n \geq 2$ is always true for $\gamma<7 / 8)$. For $m(m+2) \geq 2 /[n(1-\gamma)]$, we evaluate $\Pi^{u}(m, n)-$ $\Pi^{u}(m / 2, n / 2)$ at $t=\bar{t}(n, \gamma)$ and establish that it is always negative. We have thus proved that the set of parameters where upstream firms prefer global concentration to equal split is included in $S$.

(4) Total welfare. Total welfare is computed as $W \equiv C S+\Pi^{d}+\Pi^{u}$. Numerical simulations show that for $t>\bar{t}(n, \gamma)$, we can have $W(m, n)>$ 
$W(m / 2, n / 2)$ or $W(m, n)<W(m / 2, n / 2)$. For instance, let $m=2$ and $\gamma=0.96$. For $n=2, \bar{t}=0.00693$; taking $t=0.007$, we have $W(m, n)=$ $0.34568<W(m / 2, n / 2)=0.34862$. For $n=6, \bar{t}=0.02400$; taking $t=$ 0.025 , we have $W(m, n)=0.40916>W(m / 2, n / 2)=0.40711$.

\section{References}

[1] Alford, D., Sackett, P., Nelder, G. 2000. Mass customisation - an automotive perspective. International Journal of Production Economics 65, 99-110.

[2] Angeles, R., Nath, R. 2000. An empirical study of EDI trading partner selection criteria in customer-supplier relationships. Information \& Management 37, 241-255.

[3] Anderson, S.P., de Palma, A. 2000. From local to global competition. European Economic Review 44, 423-448.

[4] Belleflamme, P. 2000. Oligopolistic competition, IT use for product differentiation and the productivity paradox. International Journal of Industrial Organization, forthcoming.

[5] Church, J., Gandal, N. 1992. Network effects, software provision, and standardization. Journal of Industrial Economics 40, 85-103.

[6] d'Aspremont, J., Gabszewicz, J., Thisse, J.-F. 1979. On Hotelling's stability in competition. Econometrica 47, 1145-1150.

[7] Eaton, B.C., Schmitt, N. 1994. Flexible manufacturing and market structure. American Economic Review 84, 875-888.

[8] Economides, N. 1989. Desirability of compatibility in the absence of network externalities. American Economic Review 79, 1165-1181.

[9] Einhorn, M. (1992). Mix and match compatibility with vertical product dimensions. RAND Journal of Economics 23, 535-547.

[10] Fisher, M. 1997. What is the right supply chain for your product? Harvard Business Review (March-April), 105-116. 
[11] Helpman, E., Krugman, P.R., 1985. Market structure and foreign trade, MIT Press, Cambridge.

[12] Hotelling, H. 1929. Stability in competition. Economic Journal 39, 4157.

[13] Lambertini, L., Rossini, G. 1998. Product homogeneity as a prisoner's dilemma in a duopoly with R\&D. Economics Letters 58, 297-301.

[14] Levitt, T. 1980. Marketing success through differentiation-of anything. Harvard Business Review (January-February), 83-91.

[15] Marcussen, C. 1996. The effects of EDI on industrial buyer-seller relationships: a network perspective. International Journal of Purchasing and Materials Management 32, 20-26.

[16] Marshall, A., 1920. Principles of economics, Macmillan, London.

[17] Matutes, C., Régibeau, P. 1988. "Mix and match": product compatibility without network externalities. RAND Journal of Economics 19, 221-234.

[18] Matutes, C., Régibeau, P. 1989. Standardization across markets and entry. Journal of Industrial Economics 37, 359-371.

[19] Matutes, C., Régibeau, P. 1992. Compatibility and bundling of complementary goods in a duopoly. Journal of Industrial Economics 40, $37-53$.

[20] Norman, G., Thisse, J.-F. 1999. Technology choice and market structure: Strategic aspects of flexible manufacturing. Journal of Industrial Economics 47, 345-72.

[21] Rotemberg, J., Saloner, G. 2000. Competition and human capital accumulation: a theory of interregional specialization and trade. Regional Science and Urban Economics 30, 373-404.

[22] Shubik, M. 1980. Market structure and behavior. Harvard University Press, Cambridge, MA.

[23] Singh, N., Vives, X. 1984. Price and quantity competition in a differentiated duopoly. Rand Journal of Economics 15, 546-54. 
[24] Tirole, J. 1988. The theory of industrial organization. MIT Press, Cambridge, MA.

[25] Venables, A.J. 1996. Equilibrium locations of vertically linked industries. International Economic Review 37, 341-359. 


\section{Q Queen Mary \\ University of London}

This working paper has been produced by the Department of Economics at Queen Mary, University of London

Copyright ( 2000 Paul Belleflamme and Eric Toulemonde All rights reserved.

\section{Department of Economics}

Queen Mary, University of London

Mile End Road

London E1 4NS

Tel: +44 (0)20 78825096 or Fax: +44 (0)20 89833580

Email: j.conner@qmw.ac.uk

Website: www.econ.qmw.ac.uk/papers/wp.htm 